\title{
Association between $\beta$-blocker therapy and outcomes in patients hospitalised with acute exacerbations of chronic obstructive lung disease with underlying ischaemic heart disease, heart failure or hypertension
}

\author{
Mihaela S Stefan, 1,2,3,4 Michael B Rothberg, ${ }^{1,2,3}$ Aruna Priya, ${ }^{5}$ Penelope S Pekow, ${ }^{4,5}$ \\ David $\mathrm{H} \mathrm{Au}{ }^{6}$ Peter $\mathrm{K}$ Lindenauer ${ }^{1,2,4}$
}

1 Division of General Medicine Department of Medicine, Baystate Medical Center, Springfield, Massachusetts, USA

${ }^{2}$ Tufts University School of Medicine, Boston Massachusetts, USA

${ }^{3}$ Program in Clinical and Translational Research, Sackler School of Graduate Biomedical Sciences, Tufts University, Boston, Massachusetts, USA ${ }^{4}$ Center for Quality of Care Research, Baystate Medical Center, Springfield, Massachusetts, USA

${ }^{5}$ School of Public Health and Health Sciences, University of Massachusetts-Amherst, Massachusetts, USA

${ }^{6}$ Pulmonary and Critical Care Division, University of Washington, Seattle, Washington, USA

\section{Correspondence to} Dr Mihaela S Stefan, Baystate Medical Center, 756 Chestnut Street, Springfield, MA 01199 USA; mihaela.stefan@bhs.org

Received 21 March 2012 Accepted 19 July 2012

Published Online First 31 August 2012

\begin{abstract}
Background $\beta$-Blocker therapy has been shown to improve survival among patients with ischaemic heart disease (IHD) and congestive heart failure (CHF) and is underused among patients with chronic obstructive pulmonary disease (COPD). Evidence regarding the optimal use of $\beta$-blocker therapy during an acute exacerbation of COPD is particularly weak.

Methods We conducted a retrospective cohort study of patients aged $\geq 40$ years with IHD, CHF or hypertension who were hospitalised for an acute exacerbation of COPD from 1 January 2006 to 1 December 2007 at 404 acute care hospitals throughout the USA. We examined the association between $\beta$-blocker therapy and in-hospital mortality, initiation of mechanical ventilation after day 2 of hospitalisation, 30-day all-cause readmission and length of stay.

Results Of 35082 patients who met the inclusion criteria, $29 \%$ were treated with $\beta$ blockers in the first two hospital days, including $22 \%$ with $\beta 1$-selective and $7 \%$ with non-selective $\beta$ blockers. In a propensitymatched analysis, there was no association between $\beta$-blocker therapy and in-hospital mortality (OR 0.88 , $95 \% \mathrm{Cl} 0.71$ to 1.09 ), 30-day readmission (OR 0.96, 95\% $\mathrm{Cl} 0.89$ to 1.03 ) or late mechanical ventilation (OR 0.98 , $95 \% \mathrm{Cl} 0.77$ to 1.24). However, when compared with $\beta 1$ selective $\beta$ blockers, receipt of non-selective $\beta$ blockers was associated with an increased risk of 30-day readmission (OR 1.25, 95\% Cl 1.08 to 1.44).

Conclusions Among patients with IHD, CHF or hypertension, continuing $\beta 1$-selective $\beta$ blockers during hospitalisation for COPD appears to be safe. Until additional evidence becomes available, $\beta 1$-selective $\beta$ blockers may be superior to treatment with a non-selective $\beta$ blocker.
\end{abstract}

\section{BACKGROUND}

Chronic obstructive pulmonary disease (COPD) is the third leading cause of death in the USA and acute exacerbations result in more than 800000 hospitalisations annually. ${ }^{1}{ }^{2}$

The combination of COPD treatment often coexists with treatment for congestive heart failure (CHF) or ischaemic heart disease (IHD) and presents complex therapeutic challenges. $\beta 2$ Agonists,
Key messages

What is the key question?

- In patients hospitalised with acute exacerbation of chronic obstructive pulmonary disease and coexistent ischaemic heart disease (IHD) or congestive heart failure (CHF) is the receipt of a $\beta$ blocker associated with better outcomes and does the cardioselectivity of the $\beta$ blocker matter?

What is the bottom line?

- There was no association between $\beta$-blocker therapy and in-hospital mortality, 30-day readmission or late mechanical ventilation. However when compared with $\beta 1$-selective $\beta$ blockers, receipt of non-selective $\beta$ blockers was associated with an increased risk of 30-day readmission.

Why read on?

- Patients with COPD often have CHF or IHD and this combination presents complex therapeutic challenges. Continuing $\beta 1$-selective $\beta$ blockers during hospitalisation for acute COPD exacerbation appears to be safe.

which are frequently used in the treatment of COPD, have the potential for adverse cardiovascular effects including ischaemic events and arrhythmias. $^{3-5} \quad \beta$-Blocker therapy improves symptoms and survival among patients with IHD and $\mathrm{CHF}^{6}$ but is frequently withheld in patients with COPD because of concerns that it may diminish the bronchodilator effect of $\beta 2$ agonists and aggravate bronchospastic symptoms. ${ }^{8}$ Although a Cochrane meta-analysis concluded that $\beta 1$-selective $\beta$ blockade was safe, ${ }^{10} 11$ patients with COPD have half the odds of being treated with $\beta$ blockers during acute myocardial infarction (MI) than those without $\mathrm{COPD}^{12}$ and only $35 \%$ of patients with $\mathrm{CHF}$ and COPD receive $\beta$-blocker therapy. ${ }^{13} 14$ Physicians may be even more reluctant to continue $\beta$-blocker therapy during an acute 
exacerbation of COPD (AE-COPD) when a patient's respiratory status is most tenuous.

During an AE-COPD, patients may be particularly vulnerable to develop acute cardiac events ${ }^{15}$ due to increased use in $\beta 2$ agonists, tachycardia and hypoxaemia. The addition of a cardioselective $\beta$ blocker may have a cardioprotective effect in this population, blunting the cardiac toxicity of the $\beta 2$ agonists. However, information about the effectiveness and safety of $\beta$ blockers is particularly limited for patients hospitalised with AE-COPD who are treated with $\beta 2$ agonists.

To address this uncertainty, we evaluated the association of $\beta$-blocker treatment with outcomes among a large cohort of patients with IHD or CHF who were hospitalised for an AE-COPD. As a control, we also included patients with hypertension (HTN), in whom we expected to find no mortality benefit from $\beta$ blockers. Hypothesising that the $\beta 1$ selectivity of the $\beta$ blocker is associated with the severity of adverse respiratory effects, we also compared the outcomes of patients treated with a $\beta 1$-selective $\beta$ blocker with those who were treated with non-selective $\beta$ blockers.

\section{METHODS}

\section{Data source, setting and patients}

We conducted a retrospective cohort study using data collected from 404 hospitals that participate in Perspective (Premier Inc., Charlotte, North Carolina, USA), an inpatient administrative database. This dataset was described in detail elsewhere. ${ }^{16}$ Premier's Perspective data warehouse provides detailed information about all medications dispensed, laboratory tests ordered and procedures, capturing patients' complete billing and coding history during their hospital admission. It contains physician and hospital characteristics and data available from standard hospital claims: demographics, principal and secondary diagnosis and procedures, discharge status and source of admission. Hospitals are representative of all regions of the USA and are mostly small to medium size, urban and non-teaching facilities.

We included patients 40 years or older (because younger patients may have asthma not COPD), discharged from a Premier hospital between 1 January 2006 and 1 December 2007, with a principal diagnosis of AE-COPD as defined by the International Classification of Disease, Ninth Revision, Clinical Modification (ICD-9-CM; procedure codes 491.21, 491.22) or emphysema (ICD-9-CM code 492.8) or a principal diagnosis of respiratory failure (ICD-9-CM codes 518.81, 518.82, 518.84) and a secondary diagnosis of AE-COPD or emphysema. We restricted our analysis to patients with a secondary diagnosis of IHD or $\mathrm{HF}$, patients for whom $\beta$-blocker treatment may be beneficial. We also included patients with HTN as a control group. Our initial hypothesis was that receipt of a $\beta$ blocker would be associated with reduced mortality in patients with IHD and CHF but not in patients with HTN only, since there is no evidence to suggest that $\beta$-blocker therapy improves mortality in HTN, other than through the general control of high blood pressure. To increase the likelihood that the patients identified on the basis of ICD-9-CM codes were being treated for an exacerbation of COPD, we limited the analysis to those who received treatment with inhaled $\beta 2$ agonists and systemic corticosteroids on the first or second day of the hospitalisation. Patients on mechanical ventilation and those admitted directly to an intensive care unit were excluded because $\beta$ blockers may have been discontinued since clinicians may be particularly hesitant to use $\beta$ blockers in patients with severe respiratory decompensation. We also excluded patients treated with large doses of intravenous diuretics because this generally reflects treatment of acute CHF exacerbation. In addition, we excluded patients who were hospitalised for $<3$ days, because late mechanical ventilation was assessed after day 2 , patients who survived $<3$ days, and patients who were transferred from or to another facility. If a patient had more than one eligible admission during the period of the study we randomly selected one to avoid survival bias.

\section{Treatment with $\boldsymbol{\beta}$ blockers}

Receipt of a $\beta$ blocker was defined as at least one dose administered orally during any of the first two hospital days. We excluded from the analysis patients who received intravenous $\beta$ blockers because this may represent acute treatment for an arrhythmia or ischaemic event. Patients who had $\beta$-blocker treatment initiated on hospital day 3 or later were grouped with those who did not receive a $\beta$ blocker during hospitalisation because late treatment may be a marker for an acute cardiac ischaemic or arrhythmic event.

\section{Other treatments and factors}

In addition to patient demographics, primary payer and principal diagnosis, we classified comorbidities using the Healthcare Cost and Utilisation Project Comorbidity Software, V.3.1 and 3.2. ${ }^{17}$ We examined receipt of a broad range of therapies and diagnostic tests on the first 2 days of hospitalisation, including medications frequently used for AE-COPD (eg, anticholinergic bronchodilators, long-acting $\beta$ agonists) and medications frequently used for cardiovascular disease (eg, angiotensinconverting enzyme inhibitors, aldosterone antagonists, digoxin, antiplatelets, calcium channel blockers, warfarin), non-invasive ventilation, arterial blood gas and brain natriuretic peptide test. We defined early therapy as treatments in the first 2 days of hospitalisation. For each hospital, we recorded bed count, teaching status, geographic region, and whether the institution served an urban or rural population.

\section{Mortality and other outcomes}

The primary outcome was in-hospital mortality. Secondary outcomes included late mechanical ventilation (defined as invasive mechanical ventilation started on day 3 or later), length of stay and 30-day all-cause readmission. We also recorded acute ischaemic cardiac events during hospitalisation, defined as secondary diagnosis of acute MI, intermediate coronary syndrome or any receipt of therapies or procedures commonly used for the management of an acute cardiac event (glycoprotein Ilb/IIIa inhibitors thrombolytics, angiography, cardiac revascularisation) started after hospital day 2 .

\section{Analysis}

We initially used univariate and bivariate comparisons using the appropriate parametric and non-parametric tests. We developed a series of multivariable predictive models as a function of patient, treatment and hospital characteristics. Logit link functions were used for the outcomes of mortality, late ventilation and 30-day readmission and the identity link for log transformed length of stay. Generalised estimating equation models were used to account for clustering of patients within hospitals.

To further reduce the threat of selection bias (patients with the most severe AE-COPD clinical presentations might be those least likely to receive $\beta$ blockers), we developed a propensity model in which early $\beta$-blocker treatment was considered the outcome, and included all patient characteristics, early treatments and diagnostic tests, comorbidities, hospital 
characteristics and selected interaction terms as independent variables. Unadjusted, covariate adjusted and propensity score adjusted models were evaluated. In addition, we matched patients who received treatment with $\beta$ blockers with those with similar propensity who did not receive early $\beta$-blocker therapy. The propensity matched analysis was considered the primary analysis.

To further address the threat of confounding by indication we also applied an instrumental variable analysis (IVA). We used a grouped-treatment approach, a form of IVA, in which all patients treated at the same hospital are assigned a probability of treatment with a $\beta$ blocker equal to the overall treatment rate at that hospital-the grouped rate. ${ }^{18}$ This grouped rate was substituted for individual patient exposure to treatment in the logistic regression model.

To assess for heterogeneity in the association between $\beta$-blocker therapy and outcome according to the type of heart disease we tested for interactions between $\beta$-blocker therapy and HTN, IHD and CHF. In a final set of analysis, we developed a new propensity model among $\beta$-blocker treated patients only for the probability of being treated with a non-selective $\beta$ blocker. We used propensity score matching to compare the outcomes associated with the choice of $\beta 1$-selective or nonselective $\beta$ blockers. All analyses were performed using SAS V.9.2.

The institutional review board at Baystate Medical Center reviewed and approved the study protocol.

\section{RESULTS}

We identified 35082 patients who met all the inclusion criteria (figure 1). The median age was 72 years, $59 \%$ were women, $75 \%$ were white and AE-COPD was the principal diagnosis in $87 \%$ of cases (table 1). Among the entire cohort, $43 \%$ had HTN, $26 \%$ IHD with or without HTN, $31 \%$ had CHF with or without IHD or HTN and $11 \%$ had a history of MI.

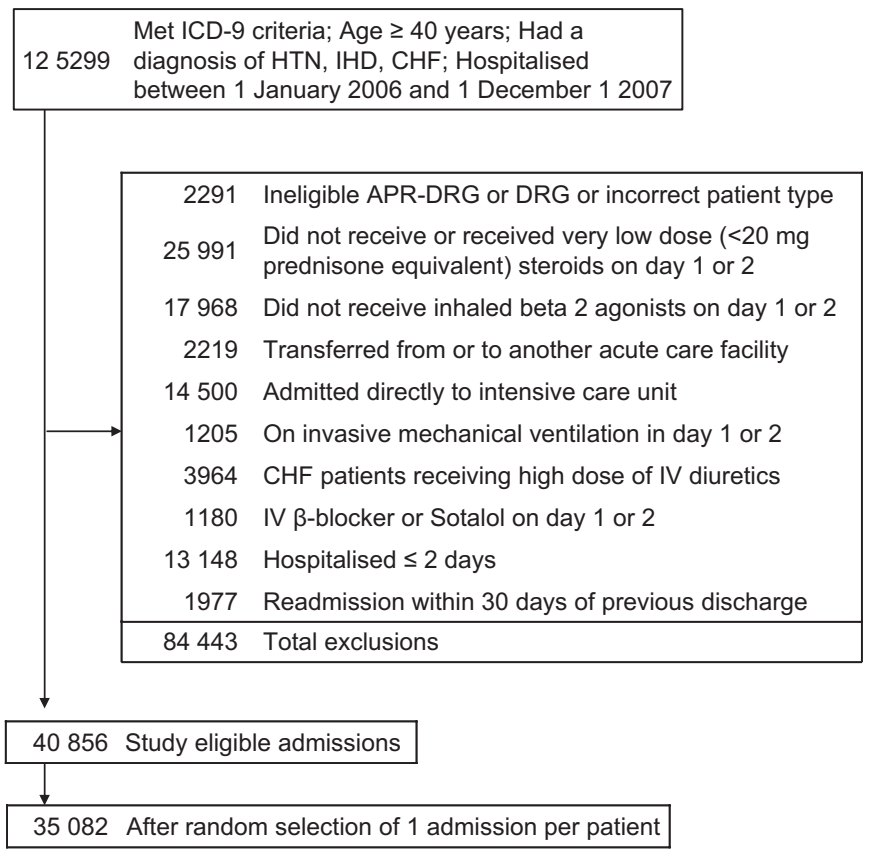

Figure 1 Patient selection flow chart. CHF, congestive heart failure; HTN, hypertension; ICD-9, International Classification of Disease, Ninth Revision; IHD, ischaemic heart disease.
A total of 10070 patients (29\%) received $\beta$ blockers within the first 2 hospital days, including $22 \%$ who were treated with cardioselective and $7 \%$ with non-selective $\beta$ blockers. There were 1379 patients who received a $\beta$ blocker starting on day 3 or later and were analysed in the group of patients who did not receive early $\beta$-blocker therapy. The most commonly used $\beta 1$-selective $\beta$ blockers were metoprolol (74\%) and atenolol (23.5\%) and nonselective $\beta$ blockers were carvedilol ( $85 \%)$ and propranolol $(7.2 \%)$. Among the 8974 patients with a diagnosis of $\mathrm{IHD}, 33 \%$ were treated with $\beta$ blockers, $82 \%$ of which were $\beta 1$ selective; among the 10921 with a diagnosis of CHF, $37 \%$ were treated with $\beta$ blockers, $64 \%$ of which were $\beta 1$ selective. Across the 404 hospitals the median rate of early use of oral $\beta$-blocker treatment was $27 \%$.

Compared with patients who were not treated with a $\beta$ blocker during the first 2 days, treated patients were older, less likely to have a primary diagnosis of respiratory failure and had fewer COPD admissions in the prior year. They had a higher prevalence of comorbidities including diabetes, atrial fibrillation, renal failure and peripheral vascular disease, were more likely to be treated with loop diuretics, digoxin, statins, antiplatelets and warfarin, but less likely to receive treatment with methylxanthines, noninvasive ventilation or to undergo an arterial blood gas analysis (table 1).

A total of 197 patients (2\%) who received early treatment with $\beta$ blockers died during hospitalisation and 191 patients (1.9\%) had mechanical ventilation initiated after day 2 of hospitalisation whereas 620 patients $(2.5 \%)$ from the untreated group died and 543 patients $(2.2 \%)$ experienced late mechanical ventilation. In total, 865 patients had an acute coronary event during hospitalisation, including $3.2 \%$ of those who received early $\beta$-blocker treatment and $2.2 \%$ of those untreated (table 2 ).

\section{Multivariable analyses}

In the analysis accounting only for within hospital clustering patients treated with $\beta$ blockers had an OR of mortality of 0.79 (95\% CI 0.67 to 0.93 ) compared with patients not treated with $\beta$ blockers. Adjustment for covariate attenuated the apparent effect of $\beta$-blocker therapy by a small amount 0.83 (0.70 to 0.99 ) and the covariate and propensity score adjusted model changed the estimate to 0.84 (0.70 to 1.00). Overall 9161 (90.8\%) of the patients treated with $\beta$ blockers within the first two hospital days were successfully matched to patients with a similar propensity score who did not receive treatment by day 2. All patient, treatment and hospital covariates were fully balanced (table 1). In this matched cohort, the effect of $\beta$ blockers on in-hospital mortality was attenuated and no longer significant (OR $0.88,95 \%$ CI 0.71 to 1.09 ).

In the multivariable and propensity matched analyses, receipt of $\beta$ blockers was not associated with late ventilation or all-cause readmission within 30 days (figure 2). Development of acute coronary events during hospitalisation (OR 1.08, 95\% CI 0.97 to 1.20) and length of stay were also not different between the two groups (OR 0.99, 95\% CI 0.994 to 0.995 ).

Interaction between early $\beta$-blocker treatment and type of cardiovascular disease (HTN, IHD and HF) was assessed in multivariable adjusted outcome models. Interaction was nonsignificant in mortality ( $p$ value $=0.9$ ), late ventilation ( $p$ value $=0.7$ ) and all-cause readmission (among survivors) ( $p$ value $=0.5$ ) models.

In the sensitivity analysis which used the hospital $\beta$-blocker prescribing rate as a form of instrumental variable, the risk of in-hospital death was not significantly different between the treated and untreated groups (OR $0.95,95 \%$ CI 0.33 to 2.72 ), but treatment with $\beta$ blockers was associated with an increased risk 
Table 1 Selected patient characteristics, comorbidities, treatments, therapies and tests (\%)

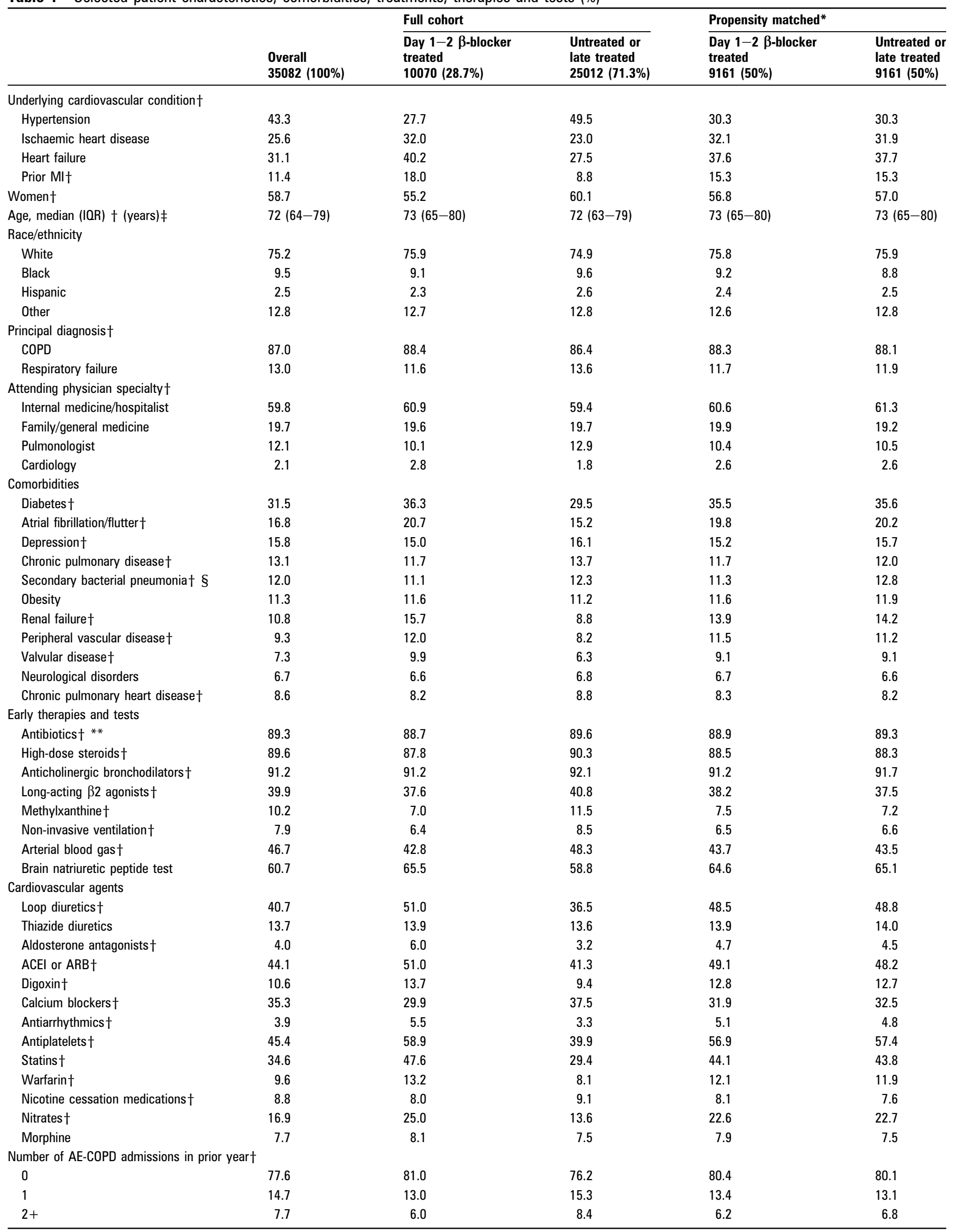


Table 1 Continued

\begin{tabular}{|c|c|c|c|c|c|}
\hline & \multirow[b]{2}{*}{$\begin{array}{l}\text { Overall } \\
35082(100 \%)\end{array}$} & \multicolumn{2}{|l|}{ Full cohort } & \multicolumn{2}{|l|}{ Propensity matched* } \\
\hline & & $\begin{array}{l}\text { Day 1-2 } \beta \text {-blocker } \\
\text { treated } \\
10070(28.7 \%)\end{array}$ & $\begin{array}{l}\text { Untreated or } \\
\text { late treated } \\
25012(71.3 \%)\end{array}$ & $\begin{array}{l}\text { Day 1-2 } \beta \text {-blocker } \\
\text { treated } \\
9161(50 \%)\end{array}$ & $\begin{array}{l}\text { Untreated or } \\
\text { late treated } \\
9161(50 \%)\end{array}$ \\
\hline \multicolumn{6}{|l|}{ Bed size† § } \\
\hline $0-200$ beds & 17.5 & 17.7 & 17.4 & 18.3 & 16.9 \\
\hline $201-300$ beds & 19.2 & 19.8 & 19.0 & 19.9 & 19.3 \\
\hline \multicolumn{6}{|l|}{ Census region $\dagger$} \\
\hline South & 53.8 & 52.3 & 54.5 & 52.9 & 53.2 \\
\hline Midwest & 17.1 & 19.3 & 16.3 & 18.7 & 18.1 \\
\hline Northeast & 16.9 & 18.1 & 16.4 & 17.8 & 17.8 \\
\hline West & 12.1 & 10.4 & 12.9 & 10.7 & 10.9 \\
\hline
\end{tabular}

*9161 of $10070(90.8 \%) \beta$-blocker treated cases matched.

tIndicates statistically significant differences between treated and untreated groups in full cohort ( $p$ value $<0.05$ )

$\ddagger$ Kruskal-Wallis tests ( $p$ value $<0.05$ ).

SIndicates statistically significant difference between treated and untreated groups in propensity-matched cohort $(p$ value $<0.05)$.

**Antibiotics included: aminoglycosides, other anti-infectives, sulfonamides, anti-MRSA medications, arbapenems, cephalosporins, macrolides, penicillins, quinolones, tetracyclines.

Additional characteristics and comorbidities evaluated in the model but not reported in the table include insurance payer, paralysis, liver disease, AIDS, lymphoma, metastatic cancer, chronic blood loss anaemia, drug abuse, pulmonary embolism, pneumothorax, polycythaemia, psychoses, alcohol abuse, solid tumour without metastasis, weight loss, rheumatoid arthritis/collagen vascular disease, sleep apnoea, pulmonary circulation disease.

ACE, angiotensin-converting enzyme inhibitors; AE-COPD, acute exacerbation of chronic obstructive pulmonary disease; ARB, angiotensin-renin blocker; COPD, chronic obstructive pulmonary disease; MI, myocardial infarction; MRSA, methicillin-resistant Staphylococcus aureus.

of late ventilation (OR $5.72,95 \%$ CI 1.47 to 22.73 ) and 30-day readmission (OR $1.50,95 \%$ CI 0.98 to 2.30 ).

\section{Cardioselective versus non-selective $\boldsymbol{\beta}$ blockers}

Overall $94.4 \%$ of patients treated with a non-selective $\beta$ blocker were matched based on propensity with a patient treated with a $\beta 1$-selective $\beta$ blocker. In this propensity-matched grouped, $\beta 1$ selectivity of the $\beta$ blocker was not associated with the risk of mortality or late ventilation. However, compared with patients treated with a $\beta 1$-selective agent, patients treated with a nonselective $\beta$ blocker had $25 \%$ increased odds of 30-day readmission (OR $1.25,95 \%$ CI 1.08 to 1.44 ) (table 3 ).

\section{DISCUSSION}

In this large observational study of more than 35000 patients hospitalised with HTN, IHD or CHF who were hospitalised for AE-COPD, treatment with $\beta$ blockers during the first 2 days of hospitalisation was not associated with the risk of in-hospital death, late mechanical ventilation, all-cause 30-day readmission or acute coronary events during hospitalisation. This pattern did not vary based on the type of heart disease or prior MI status. However, compared with treatment with $\beta 1$-selective $\beta$ blockers, therapy with non-selective $\beta$ blockers was associated with increased risk of 30-day readmission.

Among patients with a history of MI or CHF, there is clear evidence that $\beta$-blocker therapy substantially reduces mortality and morbidity. ${ }^{6}$ However, because $\beta$ blockers can accentuate bronchospastic symptoms and may diminish the bronchodilatory effects of $\beta 2$ agonists, physicians are often hesitant to treat patients with COPD with $\beta$ blockers. ${ }^{12} 14$

In this study, only $40 \%$ of patients with AE-COPD who had an evidence-based indication for chronic $\beta$-blocker therapy (an ICD-9 code of prior MI) were given a $\beta$ blocker during hospitalisation. These findings are similar to other studies that have reported the underuse of $\beta$ blockers in patients with acute $\mathrm{MI}$ or $\mathrm{CHF}$ and COPD. ${ }^{12} 14$

Table 2 Unadjusted outcomes

\begin{tabular}{|c|c|c|c|c|c|}
\hline & \multirow[b]{2}{*}{$\begin{array}{l}\text { Overall } \\
35082(100 \%) \\
\text { N (\%) }\end{array}$} & \multicolumn{2}{|l|}{ Full cohort } & \multicolumn{2}{|l|}{ Propensity matched $^{*}$} \\
\hline & & $\begin{array}{l}\text { Day } 1-2 \beta \text {-blocker } \\
\text { treated } \\
10070(28.7 \%) \\
\text { n }(\%)\end{array}$ & $\begin{array}{l}\text { Untreated or } \\
\text { late treated } \\
25012(71.3 \%) \\
n(\%)\end{array}$ & $\begin{array}{l}\text { Day } 1-2 \beta \text {-blocker } \\
\text { treated } \\
9161(50 \%) \\
n(\%)\end{array}$ & $\begin{array}{l}\text { Untreated or } \\
\text { late treated } \\
9161(50 \%) \\
\mathrm{n}(\%)\end{array}$ \\
\hline In-hospital mortality $\dagger$ & $817(2.3)$ & $197(2.0)$ & $620(2.5)$ & $178(1.9)$ & $216(2.4)$ \\
\hline Late mechanical ventilation, after day 2 & $734(2.1)$ & $191(1.9)$ & $543(2.2)$ & $174(1.9)$ & $186(2.0)$ \\
\hline 30-day all-cause readmission $\ddagger \S$ & $6891(20.1)$ & $1992(20.2)$ & $4899(20.1)$ & $1823(20.3)$ & $1876(21.0)$ \\
\hline Acute coronary events, after day $2 \dagger$ & $865(2.5)$ & $323(3.2)$ & $542(2.2)$ & $281(3.1)$ & $259(2.8)$ \\
\hline LOS, median $($ IQR) $†$ I days** & $5(4-7)$ & $5(3-6)$ & $5(4-7)$ & $5(3-6)$ & $5(4-7)$ \\
\hline
\end{tabular}

*9161 of $10070(90.8 \%) \beta$-blocker treated cases matched.

†Indicates statistically significant differences between treated and untreated groups in full cohort ( $p$ value $<0.05$ ).

$\ddagger$ Among 34265 survivors in the full cohort.

§Among 17928 survivors in the matched cohort.

TIndicates statistically significant difference between treated and untreated groups in propensity matched cohort ( $p$ value $<0.05)$.

${ }^{* *}$ Kruskal-Wallis tests ( $p$ value $<0.05$ )

AE-COPD, acute exacerbation of chronic obstructive pulmonary disease; CHF, congestive heart failure; IHD, ischaemic heart disease; LOS, length of stay. 


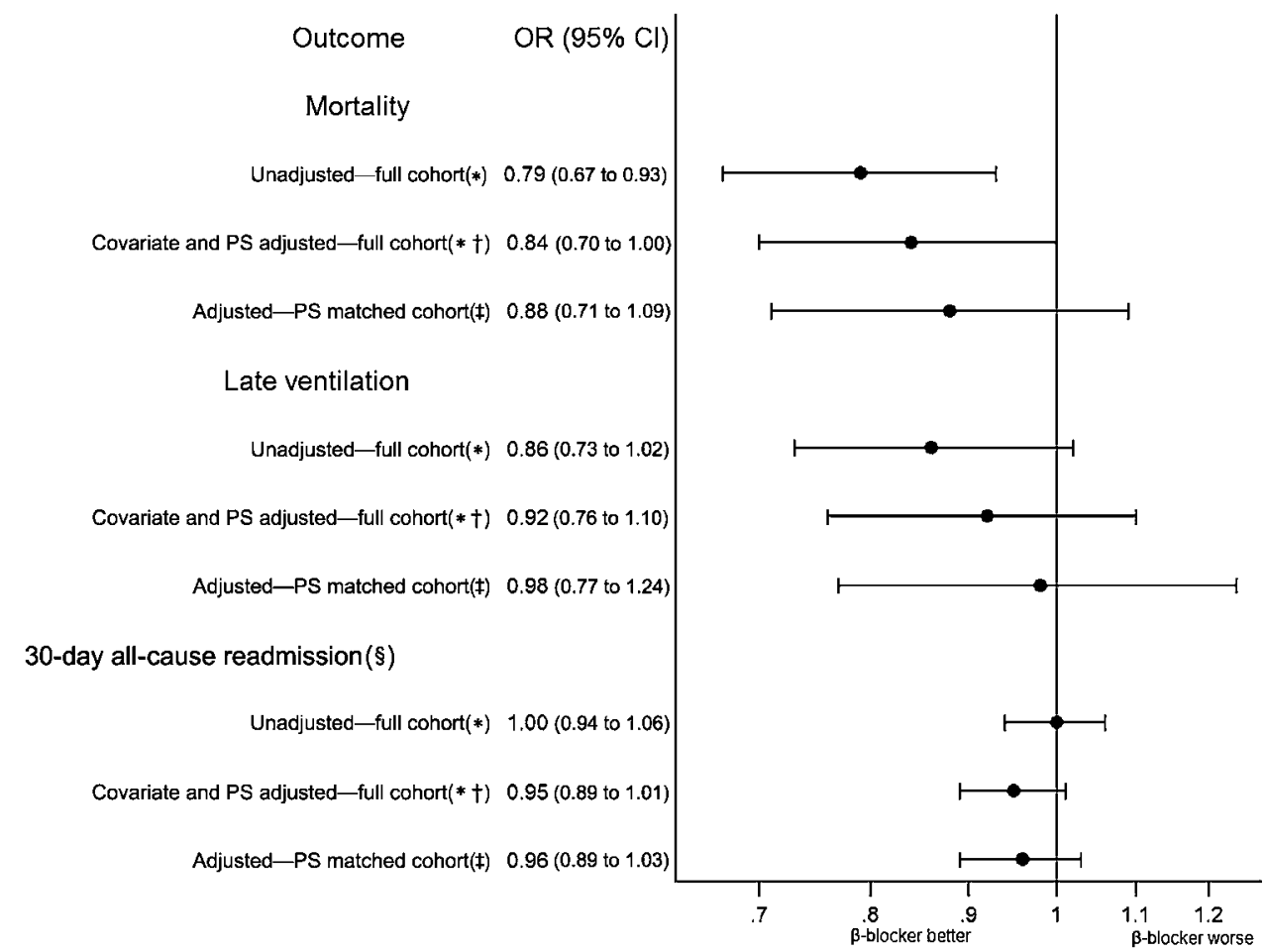

Figure 2 Outcomes of patients treated with a $\beta$ blocker on day 1 or 2 compared with late treated or untreated patients. PS, propensity score. ${ }^{*}$ Accounting for within-hospital clustering. †Covariates included in all models: underlying cardiovascular condition (hypertension (HTN), ischaemic heart disease (IHD), congestive heart failure (CHF)), age group, gender, race/ethnicity status, insurance payor, prior year admissions for chronic obstructive pulmonary disease, principal diagnosis, attending physician specialty, atrial fibrillation/flutter, valvular disease, peripheral vascular disease, other neurological disease, diabetes, hypothyroidism, renal failure, liver disease, weight loss, lymphoma, metastatic cancer, solid tumour without metastasis, deficiency anaemias, drug abuse, obesity, psychoses, depression, polycythaemia, pneumonia, pulmonary embolism, pneumothorax, other infections; and day 1 or 2 aminoglycosides, carbapenems, anti-methicillin-resistant Staphylococcus aureus (MRSA) medications, third and fourth cephalosporins, third-generation penicillins, macrolides, long-acting anticholinergic bronchodilators, methylxanthine bronchodilators, steroids, arterial blood gas, non-invasive ventilation, brain natriuretic peptide test, morphine, chronic pulmonary heart disease, loop diuretics, ace inhibitors or angiotensin II receptor blockers, calcium channel blockers, digoxin, statins, lipid lowering non-statins, antiarrhythmics, warfarin, thiazide diuretics, nicotine cessation therapy; and hospital region, bed size, rural status and selected interaction terms. ‡Covariates included underlying cardiovascular condition (HTN, IHD, CHF), secondary International Classification of Disease code for prior Ml, gender, previous year's admissions for chronic obstructive pulmonary disease, attending physician specialty, atrial fibrillation/flutter, valvular disease, peripheral vascular disease, diabetes, renal failure, lymphoma, arthritis; and day 1 or 2 long-acting anticholinergic bronchodilators, methylxanthine bronchodilators, steroids, long-acting $\beta 2$ agonists, arterial blood gas, non-invasive ventilation, chronic pulmonary heart disease, loop diuretics, angiotensin-converting enzyme inhibitors or angiotensin II receptor blockers, nitrates, digoxin, antiplatelets, calcium channel blockers, aldosterone antagonists, statins, lipid-lowering non-statins, thiazide diuretics, warfarin; and hospital region; and selected interaction terms. §Among 34265 survivors in the full cohort and 17928 survivors in the matched cohort.

Several recent observational studies have reported that longterm use of $\beta$ blockers is associated with better survival in patients with CHF or prior MI and concurrent COPD. ${ }^{19-21}$ In addition, a recent single-centre analysis of 825 patients admitted with AE-COPD ${ }^{22}$ found a $40 \%$ reduction in mortality in patients who received $\beta$-blocker therapy during hospitalisation, raising the possibility that the common practice of withholding $\beta$ blockers may be harmful. However, the very large effect estimate may have been due to immortal time bias, as well as selection of the last hospitalisation for AE-COPD, over-representing hospitalisations resulting in death. ${ }^{23}$ In contrast, our analysis suggested little if any benefit of $\beta$-blocker therapy on hospital mortality. This difference is likely due to several factors, including our selection of patients with an indication for $\beta$-blocker therapy, the large sample size from multiple hospitals, and adjustment for numerous treatments, tests and patient characteristics that could confound the relationship between $\beta$-blocker treatment and mortality. Patients with AE-COPD on $\beta$ blockers may have been those most likely to receive the therapy, that is, those with more advance cardiac disease and an increased risk of hospital deaths. Carvedilol prescription may in fact be selected for those patients who had more severe left ventricular systolic dysfunction. This idea is supported by the fact that patients receiving $\beta$-blocker therapy were more likely to be treated with 'cardiovascular' medications (loop diuretics, digoxin, statins, antiplatelets and warfarin) and had a higher prevalence of comorbidities including diabetes, renal failure and peripheral vascular disease.

There is growing evidence that $\beta 1$-selective $\beta$ blockers do not induce bronchospasm in patients with COPD. ${ }^{10} 1124$ Although there are no robust data supporting the safety of non-selective $\beta$ blockers in COPD, in our analysis $7 \%$ of all patients and $37 \%$ of patients with CHF who were treated with a $\beta$-blocker received a non-selective $\beta$ blocker, primarily carvedilol. Compared with $\beta 1$-selective agents, non-selective $\beta$ blockers were associated with an increased risk of 30-day readmission. These findings are not surprising given prior studies that have found that non-selective $\beta$ blockers increase hospitalisations in patients with COPD, decrease forced expiratory volume in $1 \mathrm{~s}$ and may inhibit bronchodilator response to $\beta 2$ agonists. 91925 Taken together our 
Table 3 Outcomes of non-selective versus cardioselective $\beta$-blocker therapy

Non-selective $\beta$ blocker versus cardioselective $\beta$ blocker*

\begin{tabular}{|c|c|}
\hline \multicolumn{2}{|l|}{ Mortality } \\
\hline Unadjusted-full cohort $†$ & $0.97(0.70$ to 1.35$)$ \\
\hline Covariate adjusted—full cohort $\ddagger$ & $0.89(0.63$ to 1.25$)$ \\
\hline Covariate and propensity score adjusted—full cohort & $0.86(0.60$ to 1.24$)$ \\
\hline Matched cohort adjusted for unbalanced covariates $\S$ ฯ & $0.75(0.47$ to 1.19$)$ \\
\hline \multicolumn{2}{|l|}{ Late ventilation } \\
\hline Unadjusted-full cohort $†$ & $1.28(0.93$ to 1.76$)$ \\
\hline Covariate adjusted—full cohort $\ddagger$ & 1.29 (0.93 to 1.79$)$ \\
\hline Covariate and propensity score adjusted—full cohort & $1.24(0.88$ to 1.74$)$ \\
\hline Matched cohort adjusted for unbalanced covariates $\S$ ฯ & $1.06(0.68$ to 1.65$)$ \\
\hline \multicolumn{2}{|l|}{ 30-Day all-cause readmission** } \\
\hline Unadjusted-full cohort $†$ & 1.35 (1.21 to 1.50$)$ \\
\hline Covariate adjusted—full cohort $\ddagger$ & 1.21 (1.08 to 1.36$)$ \\
\hline Covariate and propensity score adjusted—full cohort & $1.20(1.06$ to 1.35$)$ \\
\hline Matched cohort adjusted for unbalanced covariates $\S$ ฯ & $1.25(1.08$ to 1.44$)$ \\
\hline
\end{tabular}

*Among $\beta$-blocker treated cases $(n=10070$ ).

$\dagger$ Accounting for within-hospital clustering.

$\neq$ Covariates included in all models: underlying cardiovascular condition (hypertension, ischaemic heart disease, congestive heart failure), age group, principal diagnosis, admissions in previous year for chronic obstructive pulmonary disease, insurance payor, atrial fibrillation/flutter, pulmonary circulation disease, other neurological disorders, renal failure, metastatic cancer, solid tumour without metastasis, diabetes, weight loss, deficiency anaemias, drug abuse, psychoses, depression, sleep apnoea, pneumonia, other infections, chronic pulmonary heart disease, day 1 or 2 methylxanthine bronchodilators, arterial blood gas, non-invasive ventilation, morphine, loop diuretics, angiotensin-converting enzyme inhibitors or angiotensin II receptor blockers, antiplatelets, calcium channel blockers, lipid lowering non-statins, and hospital region.

$\S$ Each non-selective $\beta$-blocker-treated case matched on propensity with one cardioselective $\beta$-blocker-treated case. 2321 of 2459 (94.4\%) non-selective treated cases matched.

qUnbalanced covariates adjusted for attending physician specialty, valvular disease, sleep apnoea, day 1 or 2 tetracyclines, day 1 or 2 non-invasive ventilation.

**Among 9873 survivors in full cohort and 4545 survivors in matched cohort.

analyses suggest that continuing a $\beta 1$-selective $\beta$ blocker among patients who are chronic users appears to be safe during a hospitalisation for AE-COPD. However, our findings also raise additional doubts about the safety of prescribing non-selective $\beta$ blockers to patients hospitalised with COPD.

There were several limitations to this study. First, we did not have access to information about the initial gravity of COPD or the underlying severity of the cardiovascular condition, both of which might have influenced $\beta$-blocker prescribing. However, we controlled for a large number of potential confounders, including cardiovascular medications that might serve as proxies for disease severity. We also adjusted for medications frequently used for AE-COPD, prior admissions for COPD, the need for arterial blood gas testing and use of non-invasive ventilation. We attempted to overcome selection bias using several robust analytical strategies, including propensity score adjustment, matching and IVA. Second, the dataset is based on claims data and the ascertainment of comorbidities and potential contraindications is dependent on physicians' documentation and coding; however, we did not rely only on ICD-9 codes but also on daily charge files for therapies and procedures.

Third, in the sensitivity analysis, we found that higher rates of $\beta$-blocker use at the hospital level were associated with increased risk of late ventilation and 30-day readmission. Although we used IVA in an attempt to minimise confounding/ selection bias due to unmeasured factors, there was little variability of the hospital $\beta$-blocker treatment rate (median $27 \%$, IOR $22-34 \%$ ), resulting in only a weak instrument. Finally, we did not have access to ambulatory prescribing records, and therefore were unable to determine whether patients who did not receive a $\beta$ blocker in the first 2 days of hospitalisation did not receive them as outpatients or if they had their medicine withheld out of concern that this could result in further deterioration of their respiratory condition. Withholding $\beta$-blocker therapy in patients who are chronic users may be associated with an increase in mortality. ${ }^{26-28}$
In summary, our results suggest that among patients with COPD and coexistent IHD, CHF or HTN, continuing $\beta 1$-selective $\beta$-blocker therapy among chronic users appears to be safe during a hospitalisation for AE-COPD. Until additional evidence becomes available, clinicians should consider choosing $\beta 1$-selective rather than non-selective $\beta$ blockers in patients with COPD. Given the large number of patients with COPD and concurrent $\mathrm{CHF}$ and IHD, clinical trials assessing the risk-benefit of $\beta$ blockers in this population would be valuable.

Acknowledgements We thank David Kent and John Griffith from Tufts University School of Medicine for their valuable input into interpretation of the results.

Contributors MSS had full access to all of the data in the study and takes responsibility for the integrity of the data and the accuracy of the data analysis Conception and design: MSS, PSP, MBR, DHA, PKL. Analysis and interpretation: AP, MSS, PSP, MBR, PKL. Drafting the manuscript for important intellectual content: MSS, PSP, MBR, DHA, PKL.

Funding MSS is supported by KM1 CA156726 from the National Cancer Institute (NCl) and by the National Center for Research Resources and the National Center for Advancing Translational Sciences, National Institutes of Health, through grant UL1 RR025752. The content of this publication is solely the responsibility of the authors and does not represent the official views of $\mathrm{NIH}$ or NCl. The NIH or NCI had no role in the collection, management, analysis, and interpretation of the data; and preparation, review, or approval of the manuscript. DHA is supported by the Department of Veterans Affairs, Health Services Research and Development (HSR\&D). The views expressed in this article are those of the authors and do not necessarily reflect the position or policy of the Department of Veterans Affairs. DHA received grant funding from Gilead Sciences for work that is unrelated to this manuscript, and is a research consultant for Bosch LLC.

\section{Competing interests None.}

Ethics approval Ethics approval was provided by IRB of Baystate Medical Center, Springfield, Massachusetts, USA.

Provenance and peer review Not commissioned; externally peer reviewed.

\section{REFERENCES}

1. Wier LM, Elixhauser A, Pfuntner A, et al. Overview of hospitalizations among patients with COPD, 2008: statistical brief \#106. Feb 2011. In: Healthcare Cost and Utilization Project (HCUP) statistical briefs [Internet]. Rockville, MD: Agency for Health 
Care Policy and Research, USA. Feb 2006. http://www.ncbi.nlm.nih.gov.ezproxy. library.tufts.edu/books/NBK53969

2. NHLBI Morbidity and Mortality Chartbook. http://www.nhlbi.nih.gov/resources/docs/ cht-book.htm (accessed 1 Jun 2011).

3. Au DH, Curtis JR, Every NR, et al. Association between inhaled beta-agonists and the risk of unstable angina and myocardial infarction. Chest 2002:121:846-51.

4. Cazzola M, Imperatore F, Salzillo A, et al. Cardiac effects of formoterol and salmeterol in patients suffering from COPD with preexisting cardiac arrhythmias and hypoxemia. Chest 1998:114:411-15.

5. Suissa S, Hemmelgarn B, Blais L, et al. Bronchodilators and acute cardiac death. Am J Respir Crit Care Med 1996;154:1598-602.

6. Hunt SA, Abraham WT, Chin MH, et al. 2009 Focused update incorporated into the ACC/AHA 2005 guidelines for the diagnosis and management of heart failure in adults. A report of the American College of Cardiology Foundation/American Heart Association Task Force on Practice Guidelines developed in collaboration with the International Society for Heart and Lung Transplantation. J Am Coll Cardiol 2009;53:e1-90.

7. Kushner FG, Hand M, Smith SC Jr, et al. 2009 focused updates: ACC/AHA guidelines for the management of patients with ST-elevation myocardial infarction (updating the 2004 guideline and 2007 focused update) and ACC/AHA/SCAI guidelines on percutaneous coronary intervention (updating the 2005 guideline and 2007 focused update): a report of the American College of Cardiology Foundation/American Heart Association Task Force on Practice Guidelines. J Am Coll Cardiol 2009:54:2205-41.

8. Lammers JW, Folgering HT, van Herwaarden CL. Ventilatory effects of long-term treatment with pindolol and metoprolol in hypertensive patients with chronic obstructive lung disease. Br J Clin Pharmacol 1985;20:205-10.

9. van der Woude HJ, Zaagsma J, Postma DS, et al. Detrimental effects of betablockers in COPD: a concern for nonselective beta-blockers. Chest 2005:127:818-24.

10. Salpeter S, Ormiston T, Salpeter E. Cardioselective beta-blockers for chronic obstructive pulmonary disease. Cochrane Database Syst Rev 2005;(4):CD003566.

11. Salpeter SR, Ormiston TM, Salpeter EE, et al. Cardioselective beta-blockers for chronic obstructive pulmonary disease: a meta-analysis. Respir Med 2003;97:1094-101.

12. Stefan MS, Bannuru RR, Lessard D, et al. The impact of COPD on management and outcomes of patients hospitalized with acute myocardial infarction: a ten-year retrospective observational study. Chest 2012;141:1441-8.

13. Gottlieb SS, McCarter RJ, Vogel RA. Effect of beta-blockade on mortality among high-risk and low-risk patients after myocardial infarction. N Engl J Med 1998:339:489-97.
14. Egred M, Shaw S, Mohammad B, et al. Under-use of beta-blockers in patients with ischaemic heart disease and concomitant chronic obstructive pulmonary disease. OJM 2005;98:493-7.

15. Donaldson GC, Hurst JR, Smith CJ, et al. Increased risk of myocardial infarction and stroke following exacerbation of COPD. Chest 2009;137:1091-7.

16. Lindenauer PK, Pekow PS, Lahti MC et al. Association of corticosteroid dose and route of administration with risk of treatment failure in acute exacerbation of chronic obstructive pulmonary disease. JAMA 2010;303:2359-67.

17. Elixhauser A, Steiner C, Harris DR, et al. Comorbidity measures for use with administrative data. Med Care 1998;36:8-27.

18. Johnston SC, Henneman T, McCulloch CE, et al. Modeling treatment effects on binary outcomes with grouped-treatment variables and individual covariates. $\mathrm{Am} \mathrm{J}$ Epidemiol 2002;156:753-60.

19. Brooks TW, Creekmore FM, Young DC, et al. Rates of hospitalizations and emergency department visits in patients with asthma and chronic obstructive pulmonary disease taking beta-blockers. Pharmacotherapy 2007;27:684-90.

20. Rutten FH, Zuithoff NP, Hak E, et al. Beta-blockers may reduce mortality and risk of exacerbations in patients with chronic obstructive pulmonary disease. Arch Intern Med 2010;170:880-7.

21. Au DH, Bryson CL, Fan VS, et al. Beta-blockers as single-agent therapy for hypertension and the risk of mortality among patients with chronic obstructive pulmonary disease. Am J Med 2004;117:925-31.

22. Dransfield MT, Rowe SM, Johnson JE, et al. Use of beta blockers and the risk of death in hospitalised patients with acute exacerbations of COPD. Thorax 2008;63:301-5.

23. Suissa S, Ernst P. Biases in the observational study of beta blockers in COPD. Thorax 2008;63:1026-7; author reply 1027.

24. Camsari A, Arikan S, Avan C, et al. Metoprolol, a beta-1 selective blocker, can be used safely in coronary artery disease patients with chronic obstructive pulmonary disease. Heart Vessels 2003;18:188-92

25. Chang CL, Mills GD, McLachlan JD, et al. Cardio-selective and non-selective betablockers in chronic obstructive pulmonary disease: effects on bronchodilator response and exercise. Intern Med J 2009;40:193-200.

26. Psaty BM, Koepsell TD, Wagner EH, et al. The relative risk of incident coronary heart disease associated with recently stopping the use of beta-blockers. JAMA 1990;263:1653-7.

27. Teichert $\mathbf{M}$, de Smet PA, Hofman A, et al. Discontinuation of beta-blockers and the risk of myocardial infarction in the elderly. Drug Saf 2007;30:541-9.

28. Shammash JB, Trost JC, Gold JM, et al. Perioperative beta-blocker withdrawal and mortality in vascular surgical patients. Am Heart J 2001:141:148-53. 